\title{
Aspectos ecológicos de Alsophila setosa Kaulf. (Cyatheaceae, Pteridophyta) no Rio Grande do Sul, Brasil ${ }^{1}$
}

\author{
Jairo Lizandro Schmitt ${ }^{2,4}$ e Paulo Günter Windisch ${ }^{3}$
}

Recebido em 15/06/2004. Aceito em 17/05/2005

\begin{abstract}
RESUMO - (Aspectos ecológicos de Alsophila setosa Kaulf. (Cyatheaceae, Pteridophyta) no Rio Grande do Sul, Brasil). Alsophila setosa Kaulf. é uma pteridófita arborescente que ocorre em formações florestais primárias e secundárias no sul e sudeste do Brasil. O presente estudo discute a estrutura populacional de A. setosa, a arquitetura das partes subterrâneas, bem como herbivoria em suas frondes e espécies epifíticas. O trabalho de campo foi desenvolvido em duas formações vegetais secundárias, nos municípios de Morro Reuter e de Sapiranga, no Estado do Rio Grande do Sul, Brasil. Parcelas contíguas de $100 \mathrm{~m}^{2}$ foram amostradas para registro da ocorrência de A. setosa e da altura das plantas. Foram marcadas 45 plantas, em Morro Reuter, e 48 plantas, em Sapiranga, para verificar durante um ano, a ação de herbívoros em suas frondes. Cinco plantas foram desenterradas para o estudo de suas partes subterrâneas. As plantas epifíticas presentes sobre os cáudices foram registradas e amostradas. A estrutura subterrânea é complexa, com ramos caulinares que podem formar novas plantas, contribuindo para o aumento do número de indivíduos e para a distribuição espacial agregada, no local. Um grande número de plantas de menor altura foi registrado. Foram observados danos por herbivoria em $28,88 \%$ das plantas de Morro Reuter e 35,41\% das plantas de Sapiranga. Foram encontradas 16 espécies de epífitos vasculares, com predominância de holoepífitos . Os forófitos são de especial importância para a conservação de algumas espécies epifíticas.
\end{abstract}

Palavras-chave: ecologia, pteridófitas epifíticas, estrutura populacional, herbivoria, pteridófita arborescente

\begin{abstract}
Ecological aspects of Alsophila setosa Kaulf. (Cyatheaceae, Pteridophyta) in Rio Grande do Sul State, Brazil). Alsophila setosa Kaulf. is a tree fern occurring in the primary and secondary forest formations in South and Southeastern Brazil. The present study discusses population structure, architecture of the underground parts as well as herbivory of fronds and epiphytic species. Fieldwork was performed in two secondary forest tracts in the municipalities of Morro Reuter and Sapiranga in the state of Rio Grande do Sul, Brazil. Contiguous $100 \mathrm{~m}^{2}$ plots were surveyed as to the occurrence of A. setosa and plants sizes. Two samples, of 45 plants in Morro Reuter and 48 in Sapiranga, were examined throughout a full year cycle as to herbivory on their fronds. Five plants were uprooted for the study of the underground structures. The epiphytic plants on the caudexes were recorded and sampled. The underground structure is complex, with caulinar branches that may form new plants, leading to an increase of the number of individuals in a stand and to an aggregate spatial distribution. A larger number of smaller plants was recorded. Herbivory damage was observed on $28.88 \%$ of the plants in Morro Reuter and $35.41 \%$ in Sapiranga. As to vascular epiphytes, 16 species were found, with a predominance of holoepiphytes. This role as phorophyte is of special importance for the conservation of some the epiphytic species.
\end{abstract}

Key words: Ecology, epiphytic pteridophytes, population structure, herbivory, tree fern

\section{Introdução}

As pteridófitas arborescentes, geralmente conhecidas por "xaxins" ou "samambaiaçus", representam alvo de exploração extrativista, em áreas de sua maior ocorrência, especialmente no sul do Brasil. No Rio Grande do Sul, dentre as espécies exploradas está Alsophila setosa Kaulf. (Cyatheaceae) que é extraída de remanescentes florestais e utilizada para ornamentação (Windisch 2002). Além disso, as populações de $A$. setosa estão sendo reduzidas consideravelmente, em decorrência da destruição da vegetação para a realização de práticas agrícolas e da pressão de pastagem do gado, que se alimenta das frondes de plantas jovens, aumentando, consequientemente, a mortalidade das mesmas.

Existem relativamente poucos estudos abordando a ecologia de pteridófitas neotropicais. Dentre os trabalhos que discutem aspectos ecológicos de samambaias arborescentes pode-se destacar os

\footnotetext{
1 Parte da Dissertação de Mestrado do primeiro Autor

2 Centro Universitário FEEVALE, RS 239, 2755, CEP 93352-000, Novo Hamburgo, RS, Brasil

3 Programa de Pós-Graduação em Biologia, Universidade do Vale do Rio dos Sinos, São Leopoldo, RS, Brasil

4 Autor para correspondência: jairols@ feevale.br
} 
realizados por Tanner (1983), Ortega (1984), Seiler (1984), Ash (1986; 1987), Nicholson (1997), Young \& León (1989), Bittner \& Breckle (1995) e Arens \& Baracaldo (1998; 2000) que incluem informações sobre estrutura populacional de algumas espécies; Beever (1984), Rothwell (1991), Heatwole (1993), Medeiros et al. (1993), Cortez (2001), Ahmed \& Frahm (2002) e Moran et al. (2003) sobre epifitismo nos cáudices de samambaias arborescentes.

Trabalhos de taxonomia, florística e/ou fitogeografia sobre pteridófitas brasileiras que incluem Cyatheaceae são predominantemente descritivos, em geral contendo dados sucintos sobre aspectos ecológicos de Alsophila setosa. Entre esses trabalhos estão os realizados por Rosenstock (1907), Luederwaldt (1923), Sehnem (1956; 1977) e Sylvestre \& Kurtz (1994).

Além da importância florística de Alsophila setosa, seu cáudice serve de microhábitat para diversas plantas epifíticas. As informações sobre sua ecologia são escassas, sendo assim de especial importância a realização de estudos que possam fundamentar estratégias de uso, manejo e conservação, além de contribuir para a compreensão da dinâmica da vegetação onde ocorrem populações dessa espécie. Os objetivos do presente trabalho foram os de caracterizar a estrutura populacional, examinar a arquitetura das partes subterrâneas quanto à possibilidade de reprodução vegetativa, registrar a ocorrência de herbivoria e inventariar espécies epifíticas ocorrentes sobre os cáudices de A. setosa.

\section{Material e métodos}

Área de estudo - O trabalho de campo foi desenvolvido em duas formações vegetais secundárias com características de floresta estacional semidecidual, localizadas nos municípios de Morro Reuter (29³2'S e $\left.51^{\circ} 04^{\prime} \mathrm{W}\right)$, a $700 \mathrm{~m}$ alt., e de Sapiranga (29³8'S e $51^{\circ} 00^{\prime} \mathrm{W}$ ), a $570 \mathrm{~m}$ alt., no Estado do Rio Grande do Sul, Brasil. A opção por estudar a espécie em duas formações decorreu do risco de perda do material por extrativismo, durante o desenvolvimento do projeto.

Estrutura populacional - As áreas ocupadas pelas populações de Morro Reuter e de Sapiranga foram divididas, respectivamente, em sete e oito parcelas contíguas de $100 \mathrm{~m}^{2}(10 \times 10 \mathrm{~m})$. Em 2001, foi realizada a contagem de indivíduos e registrada a altura dos cáudices vivos de Alsophila setosa presentes nas parcelas. Os indivíduos foram distribuídos nas classes de altura, empregando intervalos de classe adotados por Tanner (1983): 0 a 0,8 m (Classe 1), >0,8 a 1,6 m (Classe 2), >1,6 a 2,4 $\mathrm{m}$ (Classe 3 ), $>2,4$ a $3,2 \mathrm{~m}$ (Classe 4), >3,2 a 4,0 m (Classe 5), >4,0 a $4,8 \mathrm{~m}$ (Classe 6) e $>4,8$ a 5,6 m (Classe 7). As plantas nãoférteis foram consideradas jovens e as plantas férteis, adultas. Para verificar quais classes de altura incluíam plantas jovens e adultas elaborou-se uma hipótese, baseada em observações prévias, no campo, sobre a fenologia de produção de esporos da espécie, de que existe tendência da planta tornar-se fértil (adulta) somente a partir de 2,4 m alt. Para testar essa hipótese, foi aplicada uma análise de regressão logística e o teste exato de Fischer (Zar 1999). A densidade das populações foi calculada a partir do número médio de indivíduos por $100 \mathrm{~m}^{2}$. O padrão de distribuição espacial foi determinado através do índice de Green (Ludwig \& Reynolds 1988) e da razão (R) variância/média (Brower \& Zar 1984), com significância estatística constatada, para o segundo índice, através do teste de Qui-Quadrado $\left(\chi^{2}\right)$ para um nível de significância de $5 \%$. Foi considerado como um indivíduo cada cáudice com seu conjunto de frondes.

Arquitetura subterrânea - Cinco plantas foram desenterradas para análise da arquitetura, medição do diâmetro e comprimento de suas partes subterrâneas. Foi acompanhado o desenvolvimento de uma planta jovem formada junto a um conjunto de cáudices, no período de outubro/2000 a setembro/2003, registrando a altura do cáudice e número de frondes.

Herbivoria e epifitismo - Foram selecionados 45 indivíduos de Alsophila setosa em Morro Reuter e 48 em Sapiranga, e acompanhados mensalmente, por um ano, para verificar quais deles sofreram a ação de herbívoros em suas frondes. Além disso, foram coletados, herborizados e identificados epífitos vasculares presentes sobre os cáudices dessa espécie, nos locais estudados. Os epífitos foram classificados nas categorias ecológicas propostas por Benzing (1990): holoepífitos (verdadeiros), epífitos acidentais, epífitos facultativos, hemiepífitos primários ou secundários.

Material testemunho e terminologia - O material testemunho encontra-se depositado no Herbário Pe. Aloysio Sehnem (HASU), na Universidade do Vale do Rio dos Sinos. No presente trabalho são adotados os termos cáudice, estípite e fronde, tal como definido por Lellinger (2002). As estruturas subterrâneas ramificadas ligadas à base dos cáudices foram designadas como ramificações estoloníferas. 


\section{Resultados e discussão}

Material biológico - Alsophila setosa apresenta cáudice arborescente, ereto, de até aproximadamente $10 \mathrm{~m}$ alt. com espinhos sobre as bases de estípites remanescentes das frondes caídas. Os estípites das frondes são ascendentes, com espinhos nigrescentes e curvos na porção inferior e apresentam, na porção basal, 2-4 pares de aflébias. As frondes apresentam lâmina de até cerca de $3 \mathrm{~m}$ compr., tripinatissecta, ao menos na parte basal.

Estrutura populacional - Na população de Morro Reuter, os 122 indivíduos amostrados em $700 \mathrm{~m}^{2} \mathrm{de}$ área, foram distribuídos em sete classes de altura. $\mathrm{O}$ maior cáudice registrado foi de $5,33 \mathrm{~m}$. Na população de Sapiranga, 188 indivíduos amostrados em $800 \mathrm{~m}^{2}$ de área, foram distribuídos em seis das sete classes de altura. O maior cáudice mediu 4,75 m. Com exceção da classe de indivíduos de maior altura, as duas populações apresentaram indivíduos em todas as demais classes (Fig. 1). A análise de regressão logística indicou que a fertilidade está associada à altura da planta $(\mathrm{p}=0,002)$. A categorização das plantas obtida através do teste exato de Fisher $(\mathrm{p}<0,001)$ confirmou a hipótese de que indivíduos jovens (não-férteis) apresentam geralmente até $2,4 \mathrm{~m}$ alt. e de que os adultos (férteis) são geralmente mais altos. Portanto, as classes de altura 1,2 e 3 incluem indivíduos jovens e as demais classes os indivíduos adultos.

Nos dois locais do estudo, ocorre um grande número de indivíduos de Alsophila setosa nas classes de menor

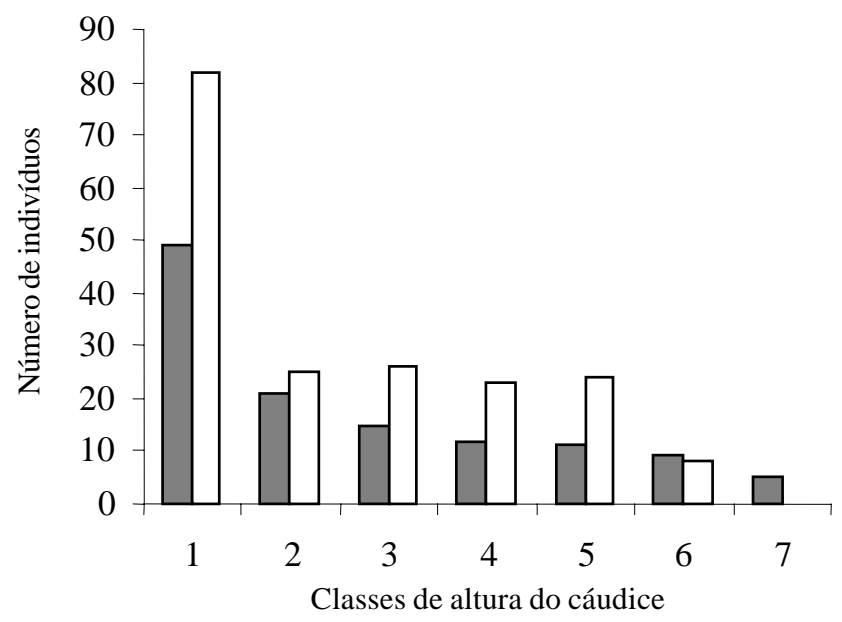

Figura 1. Distribuição de indivíduos de Alsophila setosa Kaulf. em classes de altura nas populações de Morro Reuter e de Sapiranga, RS, Brasil. Classe 1: 0 a 0,8 m; classe 2: $>0,8$ a 1,6 m; classe $3:>1,6$ a 2,4 m; classe 4: >2,4 a 3,2 m; classe 5: > 3,2 a 4,0 m; classe 6: >4,0 a 4,8 m; e classe 7: >4,8 a 5,6. $\square=$ Morro Reuter; $\square=$ Sapiranga . altura (jovens) e número menor nas classes de maior altura (adultos). Resultados similares de distribuição em classes de altura dos cáudices de pteridófitas arborescentes foram obtidos por Tanner (1983) para Cyathea pubens Mett. ex Kuhn, em montanhas da Jamaica; Ortega (1984) para uma população de Sphaeropteris senilis (Klotzsch) R.M. Tryon, crescendo no Parque Nacional El Avila da Venezuela; Seiler (1984) para Nephelea tryoniana Gastony em El Salvador; Young \& León (1989) para Trichipteris nigra (Mart.) R.M. Tryon na parte central da Amazônia peruana; e Nicholson (1997) para Nephelea cuspidata (Kunze) R.M. Tryon e Trichipteris sp. associada a Cyathea sp. na Reserva de Tambopata, Peru. Ao contrário das espécies citadas anteriormente, Leptopteris wilkesiana (Brack.) Christ apresentou uma população com poucos indivíduos jovens, em floresta primária, no Fijii, fato que pode estar relacionado a escassez de sítios com condições adequadas para o estabelecimento de indivíduos novos (Ash 1986).

Uma população estável tipicamente apresenta proporção característica de jovens, adultos jovens e adultos mais velhos. A ausência ou número baixo de jovens pode indicar que a população está declinando (Primack \& Rodrigues 2001). Nas populações de Alsophila setosa estudadas, o número de indivíduos expressivamente superior nas classes de menor altura (jovens) e um número menor nas classes de maior altura (adultos) podem indicar que as populações são estáveis ou encontram-se em fase de expansão. Young \& León (1989) sugeriram que os indivíduos mais jovens podem sofrer alta mortalidade ou supressão no crescimento e que somente poucos conseguem crescer e atingir a maturidade.

A densidade da população de Alsophila setosa em Morro Reuter foi de 17,42 $( \pm 11,54)$ indivíduos por $100 \mathrm{~m}^{2}$, sendo encontrados no mínimo um e no máximo 36 indivíduos por parcela. Na população de Sapiranga, a densidade da população foi de 23,50 $( \pm 10,81)$ indivíduos por $100 \mathrm{~m}^{2}$, sendo encontradas no mínimo nove e no máximo 38 indivíduos por parcela. A espécie apresentou padrão de distribuição espacial agregado em ambas as localidades, tanto pelo índice de Green quanto pela razão $(\mathrm{R})$ variância/média. A significância estatística foi constatada por meio dos valores encontrados de Qui-Quadrado $\left(\chi^{2}\right)$ maiores que os de tabela para os valores esperados $(\alpha=0,05)$, para o segundo índice (Tab. 1).

Sylvestre \& Kurtz (1994) citaram que em áreas bem preservadas, Alsophila setosa apresenta importância moderada na estrutura da floresta. $\mathrm{Na}$ 
Tabela 1. Distribuição espacial de Alsophila setosa Kaulf nas populações de Morro Reuter e de Sapiranga, RS, Brasil ( $\mathrm{p}<0,001 ; \alpha=0,05)$. $\mathrm{R}=$ variância/média; $\mathrm{gl}=$ graus de liberdade; $\chi^{2}=$ Qui-quadrado; $\mathrm{I} . \mathrm{G}=$ índice de Green.

\begin{tabular}{lcccccc}
\hline Local & $\mathrm{R}$ & $\mathrm{gl}$ & $\chi^{2}$ & Disribuição espacial & \multicolumn{2}{c}{ Distribuição espacial } \\
\hline Morro Reuter & $7,64^{*}$ & 6 & 45,84 & Agregado & $0,054^{* *}$ & Agregado \\
Sapiranga & $4,97^{*}$ & 7 & 34,79 & Agregado & $0,021^{* *}$ & Agregado \\
\hline
\end{tabular}

* = Resultado >1,0 indica distribuição agregada (Brower \& Zar 1984); ** = Resultado >0 indica distribuição agregada (Ludwig \& Reynolds 1988)

floresta secundária, sua presença é de grande importância, principalmente em função do elevado valor de densidade relativa, ocupando a sexta posição em relação ao índice do valor de importância. Além disso, comentaram que na região da Reserva Ecológica de Macaé de Cima, Rio de Janeiro, A. setosa encontra-se em agrupamentos de até 10 indivíduos, especialmente quando crescendo em formações secundárias. No Rio Grande do Sul, Sehnem (1956) ao observar espécimes de Alsophila setosa em seu ambiente natural, descreveu que as plantas formam pequenos bosques de degrau inferior ao dossel das florestas. I. Fernandes (dados não publicados) registrou que é comum encontrar $A$. setosa e A. sternbergii (Sternb.) Conant crescendo, em seu ambiente natural, com distribuição espacial agrupada, atribuindo o fato à reprodução vegetativa. Comentou ainda que a degradação da vegetação primária poderia oferecer a oportunidade para rápida ocupação de novos nichos, por plantas formadas a partir de ramificações estoloníferas. As observações desses autores quanto ao padrão de distribuição de A. setosa corroboram os resultados obtidos, por meio do índice de Green e da razão variância/média, no presente estudo. Arens e Baracaldo (1998) também encontraram adensamento de indivíduos de Cyathea caracasana (Klotzsch) Domin e de $C$. planadae N.C. Arens \& A.R. Sm., em floresta secundária, na Colômbia.

A distribuição agregada, tal como observada em Alsophila setosa, pode ser devida ao estabelecimento de esporófitos jovens a partir de gametófitos em nichos com condições adequadas e espaçados dentro da formação florestal, bem como ser decorrente da reprodução vegetativa. Para tentar explicar a influência da reprodução vegetativa na distribuição agregada foi feita uma verificação da arquitetura das partes subterrâneas de cinco plantas.

$O$ rizoma desenterrado de duas plantas, a profundidade de até $30 \mathrm{~cm}$, apresentou $6,4-10,5 \mathrm{~cm}$ diâm. e interligava dois cáudices próximos, distantes entre si até cerca de $60 \mathrm{~cm}$. Do rizoma partiam raízes fibrosas, que se estendiam a curtas distâncias. Rosenstock (1907) comentou que o rizoma de Alsophila setosa pode ser ricamente ramificado podendo estar tão fundo, que não é destruído com as queimadas das florestas.

$\mathrm{Na}$ base do cáudice das outras três plantas desenterradas, imediatamente abaixo à superfície do solo, foram encontradas de oito a 10 estruturas cilíndricas, aqui consideradas como ramificações estoloníferas. Essas ramificações estoloníferas apresentavam cerca de $3 \mathrm{~cm}$ diâm. sendo que algumas se estendiam além de $30 \mathrm{~cm}$, porém nenhuma delas estabelecia ligação a cáudices próximos. Por outro lado, em Sapiranga, foram encontradas duas dessas ramificações estoloníferas, descobertas pela erosão e crescendo a favor da declividade do solo, medindo aproximadamente $1 \mathrm{~m}$ compr., ligadas à base do cáudice de indivíduos próximos. Na porção terminal dessas duas ramificações estoloníferas formavam-se báculos, que se expandiam caracterizando um processo de reprodução vegetativa e o início de formação de um novo cáudice.

Em outubro/2000, na população de Morro Reuter, a cerca de $3 \mathrm{~m}$ de distância das plantas marcadas, foi encontrado um báculo emergindo do solo. Em fevereiro do ano subseqüente, no local do báculo, havia um cáudice de $7 \mathrm{~cm}$ alt. com duas frondes. Posteriormente, em abril/2001, o mesmo cáudice estava com $10 \mathrm{~cm}$ alt. e seis frondes expandidas. Em uma visita adicional, realizada em setembro/2003, o cáudice formado apresentava $70 \mathrm{~cm}$ alt. com uma coroa de frondes com 3,5 m diâm. Foi constatado pelas observações de campo que o cáudice se originou de uma ramificação estolonífera.

Assim sendo, além da planta apresentar um rizoma subterrâneo que pode interligar cáudices próximos, aparentemente são formados ramos mais esguios, que podem se estender por distâncias maiores e formar novos cáudices adiante. Considerando que nas observações de campo foram vistas várias ramificações estoloníferas que não estavam ligadas a cáudices 
próximos, é possível que eles possam se individualizar, posteriormente à decomposição das partes subterrâneas antigas, caracterizando a formação de uma nova planta. Como o esclarecimento de tal questão envolveria a destruição de grande número de plantas e impacto no local, as observações foram apenas de caráter descritivo. Desta maneira, para a análise populacional cada cáudice foi considerado como um indivíduo, tal como em geral é o caso nas ciateáceas. A questão ainda requer maiores estudos.

Mesmo que a reprodução vegetativa não exclua a possibilidade do estabelecimento de novos indivíduos a partir de gametófitos, percebeu-se que a reprodução por ramificações estoloníferas em Alsophila setosa é processo que acontece de maneira muito rápida, indicando grande potencial de multiplicação de organismos que pode ser utilizado em projetos de restauração, conservação e manejo de ambientes naturais, diminuindo as conseqüências do extrativismo da espécie.

Herbivoria - Foram observados danos causados por herbivoria nas frondes de Alsophila setosa em 28,88\% e $35,41 \%$ das plantas de Morro Reuter e de Sapiranga, respectivamente. Foi observada, porém não quantificada, preferência dos herbívoros por frondes de $A$. setosa recém-expandidas ou que se encontravam em fase de expansão. Em alguns casos, o tecido inteiro da lâmina foi consumido ou o báculo não completava seu processo de expansão em função dos prejuízos causados pela herbivoria. Embora isto pudesse ter aumentado a probabilidade de morte, reduzido a capacidade fotossintetizante, ou levado a um menor desenvolvimento das plantas, devido aos danos ao tecido laminar, não se observou perda de indivíduos nas populações pela ação dos herbívoros. A herbivoria também foi registrada para outras espécies de pteridófitas por Balick et al. (1978) que encontraram percentuais altos de danos causados nas frondes de Blechnum varians (Fourn.) C.Chr. (12\%), Polystichum muricatum (L.) Fée (16\%) e Thelypteris cheilanthoides (Kunze) Proctor (38\%); Hendrix \& Marquis (1983) que encontraram danos de herbivoria nas frondes de Thelypteris turrialbae (Rosenst.) C.V. Morton (5,5\%), Adiantum obliquum Willd. (7,3\%) e Polybotrya cervina (L.) Kaulf. (9,9\%); e Mehltreter $\&$ Tolome (2003) que encontraram danos de herbivoria em frondes de Diplazium expansum Willd. (5,8\%), Blechnum glandulosum Link (6,1\%) Marattia laxa Kunze (11,1\%), significativamente maiores em frondes jovens de que em maduras.
Durante os trabalhos de campo não foram observados os animais buscando alimento nas plantas, sendo que provavelmente os herbívoros possuam hábitos noturnos. No entanto, Luederwaldt (1923) registrou herbivoria em frondes de esporófitos de Alsophila setosa por larvas de insetos e formigas cortadeiras, como Acromyrmex nigrosetosus Forel.

Foram encontradas formigas arborícolas, do gênero Iridomyrmex, nos espécimens de Alsophila setosa das duas populações. Contudo, estas formigas não são herbívoras. Segundo Jaffé (1993), as formigas do gênero Iridomyrmex pertencem à subfamília Dolichoderinae, que inclui bom número de gêneros arborícolas. As formigas de hábito arborícola utilizam fibras vegetais para construírem seus ninhos, característica esta que pode ser considerada para explicar a presença delas, dentro das cavidades de estípites remanescentes nos cáudices de A. setosa.

Epifitismo - Foram amostradas 16 espécies de plantas epifíticas sobre os cáudices de Alsophila setosa, pertencentes a 13 gêneros, distribuídas em oito famílias botânicas. Os gêneros com maior riqueza de espécies foram Campyloneurum, Pecluma e Trichomanes com duas espécies cada; os demais gêneros apresentaram uma única espécie (Tab. 2). Analisando-se todas as espécies coletadas, observou-se a predominância de espécies de Polypodiaceae (oito espécies) e Hymenophyllaceae (duas espécies). Estas famílias foram consideradas entre as famílias epifíticas mundialmente mais ricas por Madison (1977), Kress (1986) e Benzing (1990) assim como na região Neotropical por Gentry \& Dodson (1997).

A categoria dos holoepífitos foi a mais numerosa (Tab. 2) com 12 espécies (75\%), seguida pela categoria de epífitos facultativos, com três espécies (19\%) e hemiepífitos secundários, com uma espécie (6\%). Os holoepífitos também foram mais numerosos no estudo realizado por Borgo et al. (2002) sobre indivíduos arbóreos, em floresta estacional semidecidual, no Estado do Paraná, Brasil.

Foi registrada a presença de plântulas de Araucaria angustifolia (Bert.) O. Kunze, que ocorreu, provavelmente, devido ao depósito de sementes no cáudice por algum animal, visto não haver araucárias na vizinhança imediata. Porém, esta espécie não foi incluída como epífito acidental porque não completa todo seu ciclo de vida sobre o forófito estudado. Por outro lado, não foram encontradas orquídeas sobre os cáudices nos locais estudados. Neste caso, provavelmente houve influência da ação 
Tabela 2. Famílias e espécies de epífitos vasculares nos cáudices de Alsophila setosa Kaulf. amostrados nos municípios de Morro Reuter e de Sapiranga, RS, Brasil, com a sua respectiva categoria ecológica. ${ }^{1}$ Categorias ecológicas: $\mathrm{HOL}=$ holoepífito; $\mathrm{FAC}=$ epífito facultativo; HEM = hemiepífito secundário.

\begin{tabular}{|c|c|}
\hline Família/Espécie & Cat. Ecol. ${ }^{1}$ \\
\hline \multicolumn{2}{|l|}{ ASPLENIACEAE } \\
\hline Asplenium scandicinum Kaulf. & HOL \\
\hline \multicolumn{2}{|l|}{ BLECHNACEAE } \\
\hline $\begin{array}{l}\text { Blechnum binervatum (Poir.) C.V. Morton } \\
\text { \& Lellinger }\end{array}$ & HEM \\
\hline \multicolumn{2}{|l|}{ BROMELIACEAE } \\
\hline Tillandsia aëranthos (Loisel.) L.B. Smith & HOL \\
\hline \multicolumn{2}{|l|}{ DRYOPTERIDACEAE } \\
\hline \multicolumn{2}{|l|}{ HYMENOPHYLLACEAE } \\
\hline Trichomanes angustatum Carm. & HOL \\
\hline Trichomanes radicans $\mathrm{Sw}$. & HOL \\
\hline \multicolumn{2}{|l|}{ PIPERACEAE } \\
\hline Peperomia sp. & HOL \\
\hline \multicolumn{2}{|l|}{ POLYPODIACEAE } \\
\hline $\begin{array}{l}\text { Campyloneurum austrobrasilianum (Alston) } \\
\text { de la Sota }\end{array}$ & HOL \\
\hline Campyloneurum nitidum (Kaulf.) C. Presl & FAC \\
\hline Microgramma squamulosa (Kaulf.) de la Sota & HOL \\
\hline Niphidium rufosquamatum Lellinger & FAC \\
\hline Pecluma pectinatiformis (Lindm.) M.G. Price & HOL \\
\hline Pecluma truncorum (Lindm.) M.G. Price & HOL \\
\hline Pleopeltis angusta Willd. & HOL \\
\hline Polypodium hirsutissimum Raddi & HOL \\
\hline \multicolumn{2}{|l|}{ VITTARIACEAE } \\
\hline Vittaria lineata (L.) Sm. & HOL \\
\hline
\end{tabular}

humana, pois orquídeas epifíticas podem ser observadas em plantas de outras localidades da região, em situações menos acessíveis ao extrativismo.

Luederwaldt (1923) citou Asplenium harpeodes Kunze e Trichomanes augustatum Carm. como epífitos de Alsophila setosa (como Hemitelia setosa (Kaulf.) Mett.). Sehnem (1956), citou outras espécies, entre as quais Campyloneurum phyllitidis (L.) C. Presl. (como Polypodium phyllitidis L.), Pecluma truncorum (Lindm.) M.G. Price (como Polypodium truncorum Lindm.), Asplenium harpeodes Kunze (como A. erectum Bory ex Willd.), A. scandicinum Kaulf., A. mucronatum C. Presl. e a orquidácea Zigopetalum maxillare Lodd. A presença de Asplenium scandicinum, Campyloneurum phylitidis e Blechnum binervatum (Poir.) Mort. \& Lell. crescendo sobre cáudices de A. setosa foi registrada por Sehnem (1977), na mata pluvial de Morro Reuter.

Os cáudices dos esporófitos de Alsophila setosa servem de substrato para várias plantas. O predomínio de holoepífitos, ou seja, de plantas que completam todo o seu ciclo de vida habitualmente no ambiente epifítico, destaca a importância do suporte físico oferecido pelos cáudices dessa espécie, nos remanescentes florestais. Além disso, algumas plantas epifíticas são específicas ou ocorrem preferencialmente sobre os cáudices de samambaias arborescentes, tal como Zigopetalum maxillare, Pecluma truncorum (como Polypodium truncorum), Asplenium mucronatum citadas por Sehnem (1977); bem como determinadas espécies de Hymenophyllaceae (Cortez 2001), tal como Trichomanes angustatum e $T$. radicans $\mathrm{Sw}$, encontradas no presente estudo. Cortez (2001) destacou que o substrato oferecido pelos cáudices das samambaias arborescentes apresenta, aparentemente, condições ótimas de umidade e porosidade para as espécies epifíticas. Assim, o extrativismo, com o conseqüente desaparecimento das plantas adultas de $A$. setosa, compromete a disponibilidade de hábitats específicos de outras espécies, sendo que este fato merece especial atenção no que tange à conservação de espécies.

\section{Agradecimentos}

Os autores agradecem a Julian Mauhs e à Maria A.K. Rubio por terem colaborado na localização das populações estudadas; a Renato R. Fleck e à Regina K. Veroneze, que autorizaram a realização dos trabalhos de campo em suas propriedades; a Carlos Rodrigo Lehn, Lucas Schmitt, Jorge L. Pereira, Guilherme L. Jacobs e à Cristina L. Jacobs Schmitt, pelo auxílio nos trabalhos de campo; ao Centro Universitário FEEVALE, pela concessão de auxílio financeiro; à Universidade do Vale do Rio dos Sinos, pela infra-estrutura disponibilizada; aos revisores anônimos, por sugestões encaminhadas.

\section{Referências bibliográficas}

Ahmed, J. \& Frahm, J.P. 2002. Moosgesellschaften auf baumfarnstämmen in südostbrasilien. Tropical Bryology 22: $135-178$.

Arens, N.C. \& Baracaldo, P.S. 1998. Distribution of tree ferns (Cyatheaceae) across a sucessional mosaic in an Andean cloud forest, Nariño, Colombia. American Fern Journal 88: 60-71.

Arens, N.C. \& Baracaldo, P.S. 2000. Variation in tree fern stipe length with canopy height: tracking preferred habitat through morphological change. American Fern Journal 90: 1-15. 
Ash, J. 1986. Demography and production of Leptopteris wilkesiana (Osmundaceae), a tropical tree-fern in Fiji. Australian Journal of Botany 34: 207-215.

Ash, J. 1987. Demography of Cyathea hornei (Cyatheaceae), a tropical tree-fern in Fiji. Australian Journal of Botany 35: 331-342.

Balick, M.J.; Furth, D.G. \& Cooper-Driver, G. 1978. Biochemical and evolutionary aspects of arthropod predation on ferns. Oecologia 35: 55-89.

Beever, J. 1984. Moss epiphytes of tree ferns in a warm temperature forest. New Zealand Journal Hattori Botanical Laboratory 56: 89-95.

Benzing, D.H. 1990. Vascular epiphytes. Cambridge, Cambridge University Press.

Bittner, J. \& Breckle, S.W. 1995. The growth rate and age of tree fern trunks in relation to habitats. American Fern Journal 85: 37-42.

Borgo, M.; Silva, S.M. \& Petean, M.P. 2002. Epífitos vasculares em um remanescente de floresta estacional semidecidual, município de Fênix, PR, Brasil. Acta Biológica Leopoldensia 24(2): 199-203.

Brower, J.E. \& Zar, J.H. 1984. Field \& laboratory methods for general ecology. $2^{\text {nd }}$ ed. Iowa, Wm. C. Publishers.

Cortez, L. 2001. Pteridofitas epifitas encontradas en Cyatheaceae y Dicksoniaceae de los bosques nublados de Venezuela. Gayana Botanica 58: 13-23.

Gentry, A.H. \& Dodson, C.H. 1987. Diversity and biogeography of neotropical vascular epiphytes. Annals of the Missouri Botanical Garden 74: 205-233.

Heatwole, H. 1993. Distribution of epiphytes on trunks of the arborescent fern, Blechnum palmiforme, at Gough Island, south Atlantic. Selbyana 14: 46-58.

Hendrix, S.D. \& Marquis, R.J. 1983. Herbivore damage to three tropical ferns. Biotropica 15: 108-111.

Jaffé, C.K. 1993. El mundo de las hormigas. Caracas, Editorial Equinoccio, Universidad Simon Bolivar.

Kress, W.J. 1986. The systematic distribution of vascular epiphytes: an update. Selbyana 9: 2-22.

Lellinger, D.B. 2002. A modern multilingual glossary for Taxonomic Pteridology. Pteridologia n.3. Washington, American Fern Society.

Ludwig, J.A. \& Reynolds, J.F. 1988. Statistical ecology: a primer on methods and computing. New York, J. Wiley \& Sons, USA.

Luederwaldt, von H. 1923. Die Cyathaceen aus der Umgebung der Stadat S. Paulo. São Paulo, Zeitschriff Deutscher Verein für Wissenschaft und Kunst.

Madison, M. 1977. Vascular epiphytes: their systematic occurrence and salient features. Selbyana 2: 1-13.
Medeiros, A.C.; Loope, L.L. \& Anderson, S.J. 1993. Differential colonization by epiphytes on native (Cibotium ssp.) and alien (Cyathea cooperi) tree ferns in a Hawaiian rain forest. Selbyana 14: 71-74.

Mehltreter, K. \& Tolome, J. 2003. Herbivory on three tropical fern species of a mexican cloud forest. Pp. 375-381. In: S. Chandra \& M. Srivastava (eds.). Pteridology in the New Millennium. The Netherlands, Kluwer Academic Publishers.

Moran, R.C.; Klimas, S. \& Carlsen, M. 2003. Low-trunk epiphytic ferns on tree ferns versus angiosperms in Costa Rica. Biotropica 35: 48-56.

Nicholson, B. 1997. Obervations on the distribution and diversity of tree ferns in the Zona Reservada de Tambopata, Madre Rios, Peru. Fern Gazette 15: 153-159.

Ortega, F. 1984. Notas sobre la autoecologia de Sphaeropteris senilis (K1.) Tryon (Cyatheaceae) en el Parque Nacional El Avila - Venezuela. Pittieria 12: 31-53.

Primack, R.B. \& Rodrigues, E. 2001. Biologia da conservação. Londrina, Editora Rodrigues.

Rosenstock, E. 1907. Beiträge zur Pteridophytenflora Südbrasiliens. II. Hedwigia 46: 57-69.

Rothwell, G.W. 1991. Botryopteris forensis (Botryopteridaceae), a trunk epiphyte of the tree fern Psaronius. American Journal of Botany 78: 782-788.

Sehnem, A. 1956. Uma coleção de pteridófitos do Rio Grande do Sul. Sellowia 7: 299-327.

Sehnem, A. 1977. As filicíneas do Sul do Brasil, sua distribuição geográfica, sua ecologia e suas rotas de imigração. Pesquisas Botânica 31: 1-108.

Seiler, R.L. 1984. Trunk length and frond size in a population of Nephelea tryoniana from El Salvador. American Fern Journal 74: 105-107.

Sylvestre, L.S. \& Kurtz, B.C. 1994. Cyatheaceae. Pp. 139-152. In: M.P.M. Lima \& R.R. Guedes-Bruni (orgs.). Reserva Ecológica de Macaé de Cima, Nova Friburgo-RJ. Aspectos florísticos das espécies vasculares. v.1. Ministério do Meio Ambiente e da Amazônia Legal/ IBAMA. Rio de Janeiro, Jardim Botânico do Rio de Janeiro, Rio de Janeiro.

Tanner, E.V.J. 1983. Leaf demography and growth of treefern Cyathea pubescens Mett. ex Kuhn in Jamaica. Botanical Journal of the Linnean Society 87: 213-227.

Windisch, P.G. 2002. Fern conservation in Brazil. Fern Gazette 16: 295-300.

Young, K.R. \& León, B. 1989. Pteridophyte species diversity the Central Peruvian Amazon: importance of edaphic specialization. Brittonia 41: 388-395.

Zar, J.H. 1999. Biostatistical analysis. New Jersey, Prentice Hall, Upper Saddle River. 